\title{
Moving from analog to digital driving
}

\author{
Alberto Broggi, Stefano Debattisti, Matteo Panciroli and Pier Paolo Porta \\ VisLab - Dipartimento di Ingegneria dell'Informazione \\ Università degli Studi di Parma, ITALY \\ http://www.vislab.it \\ \{broggi, deba, panci, porta\}@vislab.it
}

\begin{abstract}
This paper presents a new vehicle guidance paradigm based on high level commands together with a new system architecture that can handle different levels of automation. First, an overview of different automation levels spanning from manual driving to fully autonomous driving is given, then a detailed description of the digital driving paradigm is also shown. This includes a detailed description of the maneuver level planner which is based on a new concept of planning based on checkpoints. Then, an overview of the new architecture is given, describing is key aspects. An analysis of the proposed paradigm, with the description of a preliminary tests in real scenarios concludes this paper.
\end{abstract}

\section{INTRODUCTION}

In the last decade, intelligent systems have been integrated in all kind of vehicles. The increasing autonomy of the vehicle itself leads to a subsequent need for cooperation with the driver who is formally in charge of the driving action. In this paper different automation levels will be discussed with a particular emphasis on the maneuver level, here defined as digital driving. At this level the driver only commands maneuvers to the vehicle, i.e. a discrete set of actions, instead of analog inputs (pedals, steering wheel) [1].

The current two main research streams focusing on intelligent vehicles are Advanced Driver Assistance Systems (ADAS) and autonomous vehicles. The interest on autonomous vehicles has been high until the late 90s, when the exploitation of full automation was felt as very close but ADAS systems started attracting the attention of the major automotive companies. In the last few years a new interest in autonomous vehicles grew up, mainly based on applications oriented to particular environments such as agricultural, mining, military, exploration.

ADAS are designed mainly for predefined scenarios such as highways, parking lots, queue driving, etc. and their performance needs to be high in their domain of applicability. Reduced cost and size are also mandatory to allow straightforward integration and thus commercialization in the automotive market [2], [3]. On the other hand for autonomous vehicles, systems cost and size are currently less important but they have to deal with all weather conditions, all terrain, and all scenarios [4], [5], [6].

Even if the final aim of the system is different, most of the underlying ideas are shared -such as for example perception, planning and actuation- but the different requirements in cost and integration lead to different sensing and processing engines [7]. The main difference between the two branches

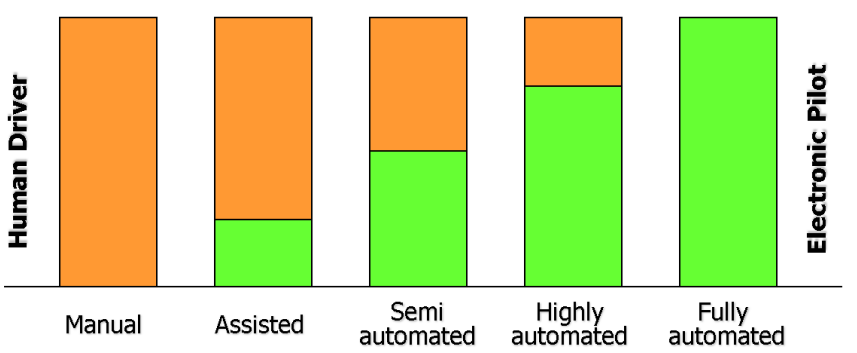

Fig. 1. Taxonomy for vehicle automation.

is definitely the presence or absence of the driver; this may also imply a difference of responsibility in case of fault.

Current basic DAS such as ABS, ESP, etc. act without the need for the driver to override the system, because they feature limited sensing, straightforward decisions and actuations, and are applicable in well defined scenarios; in these cases only a short term automation is involved. On the contrary, ADAS include complex sensing and actuation, the cost limitation does not allow to have a redundant sensing of the environment, while their applicability is again limited to specific scenarios.

Different levels of automation are possible between manual driving and fully autonomous driving as shown in figure 1. The first step after pure manual driving consists of Assisted driving, where the vehicle only issues suggestion to the driver. An example of a system included in this level is Lane Departure Warning (LDW), since no actuation on vehicle control but only an acoustical or haptic feedback to the driver is given.

Semi automated level is characterized by the automation of selected tasks to help the driver in a predefined set of situations and it involves only a quick intervention on actuators to correct and recover from potentially dangerous situations. A Lane Keeping Assistant (LKA) is part of this level; the driver still acts on vehicle control, but sometimes he is helped by the intelligent vehicle. Automatic Cruise Control (ACC) belongs to this level too: the car keeps a safe distance from the preceding vehicle when the system is engaged.

In highly automated level of automation the vehicle can make maneuvers and hold the control even for a long time in specific scenarios. In this case for example, the car and the driver meet at maneuver level. An example of this level can be LKA+ACC: the vehicle is able to keep a safe speed and a 
correct distance to the vehicle ahead, and follow the road. It involves lateral and longitudinal control with full autonomy in a specific and limited scenario (highway environment, in this case). The last step is fully autonomous driving. The driver only defines the destination, while all the driving action, including planning, is left to the intelligent vehicle.

Regardless of the state of the research in intelligent vehicles, it is not improbable that current autonomous driving systems are affected by errors, in particular related to the perception of the surroundings. Managing perception errors is a key point during autonomous system design and recovery could be difficult, especially in case of false negatives with respect to false positives. For this reason, conservative policies are often preferred in order to bias the system through false positives instead false negatives. Moreover recovering from a perception error could be computationally heavy and time consuming [8]. Keeping the driver in the loop could solve most of these problems and reduce the time for error recovery since a human driver can easily recognize false positives and, at the same time, manage emergencies quickly choosing the best solution [9]. Moreover, according to the Wien 1968 road traffic convention [10], each vehicle needs a driver that hold the responsibility in case of accident, even if during autonomous driving session; thus driver supervision is made mandatory since these systems are designed to complement the driver and not to replace it.

The increasing number of ADAS present on highly automated vehicles could pose a further problem: these systems are often heterogeneous and independent one from the other, leading to an overload for the driver who is required to supervise all of them with a consequent increased difficulty in the driving action.

Given all the problems described, a new highly automated driving paradigm, called Digital Driving, has been developed.

\section{Digital Driving}

In literature different solutions are present to solve the problems described in the previous section. In [11] a drivervehicle cooperation method called H-mode is presented; the approach follows the horse metaphor. The purpose of this approach is to develop an haptic-multimodal interaction in the field of semiautonomous driving which enables the system to suggest specific actions (e. g. a lane change) to the driver. Another solution is the one presented by TU Darmstad based on the Conduct By Wire paradigm: a discrete set of elements, called maneuvers, are used to drive the vehicle. Each maneuver, e.g. lane change, is directly translated into actions by the vehicle without any intervention on traditional interfaces such as steering wheel or pedals by the driver [12].

The approach proposed in this paper, called Digital Driving, is based on maneuvers as well but with an higher automation grade; plus, potential conflicts between driver and vehicle are taken into account and managed [13].

In [14], the driving action is divided in three different levels: navigation, guidance (or maneuver), and stabilization. At navigation level the driver chooses an appropriate path to reach his/her destination; this task is typically done before departure and could be reissued in case of problems during the trip like road closure. The guidance level, also called maneuver level, includes the subjective generation of all the trajectories needed to drive along the chosen road; these actions do not affect the navigation plan decided at the previous level. The last and lowest level, called stabilization level, is about the correct placement of the vehicle in the road; the local position refinement is done by the driver by means of steering wheel, gas, and brake.

In traditional cars a driver has to perform simultaneously on all the three levels: starting from planning a trip and alternative routes (navigation level), he then chooses an adequate trajectory and speed or he takes other decisions like lane changes (maneuver level), which he finally transforms into concrete actions, like e.g. steering or braking (stabilization level). Even in presence of ADAS, these systems work mainly on separated levels and there is no cooperation between them. Therefore, the goal of the Digital Driving consists of promoting the car-driver interaction from the stabilization level to the maneuver level, thus allowing the driver to go beyond the direct control of the vehicle through the traditional interfaces used in the stabilization level.

Among the main advantages of this approach, in this case the human driver is excluded from the stabilization tasks, thus allowing a safe reaching of the destination even in case of distracted or fatigued driver. Another point is that with this paradigm also less skilled drivers could be able to drive. Thanks to a reduced set of maneuvers, detailed in section II-A, just one of them will be selected, as for example "Highway driving" without defining how to perform this action. During the design and implementation of this paradigm the human driver has been kept on the perceptionaction loop in order to establish a cooperation and to keep him responsible for the driving action. This has been possible thanks to the management of possible conflicts implementing a conflict resolution policy.

The three main elements of Digital Driving are:

- Human-Machine Interface: it eases the driver-vehicle interaction, as a good trade off between efficiency and comfort;

- Maneuver Catalogue: it defines all possible actions that can be issued by the driver to the vehicle;

- Conflict Resolution Policy: a conflict resolution policy has to be implemented in order to take advantage of the driver presence on board.

This paper is focused on the maneuvers catalogue and on his implementation.

\section{A. Maneuver Catalogue}

Part of the work made for the implementation of the Digital Driving paradigm has been spent in the creation of a maneuver catalogue that must be complete and compact, in order to cover all possible cases that the driving action can generate. In this paradigm, a driver must have a perfect knowledge about the maneuvers catalogue, thus the number of possible maneuvers is a key issue, as explained in [15]. In the literature different approaches have been highlighted: 
[16] performs an analytic analysis of all the driving situations and distinguishes more than 3 million possible maneuvers, while in [15] a more simple approach is used and the catalogue is composed by only 17 maneuvers.

To better address the problem the maneuver set has been analyzed and divided in 2 layers. The first layer is composed by high levels goals called behaviors, while the second one is composed by maneuvers useful to reach the final goal, called sub-maneuver. Let us consider for example the behavior "Highway driving"; the goal could be reached with different maneuvers: lane keeping, vehicle following, overtaking, depending on local conditions. The driver has thus only to select a behavior while the maneuver level planner will choose the proper sub-maneuver to accomplish the task. Behaviors are related to the inner goal of an action and are at a higher hierarchical level than the sub-maneuvers, as shown int the architecture representation of figure 3 . Furthermore, each behavior is composed by many different maneuvers, exactly as the "Highway Driving" behavior is composed by a list of sub-maneuvers that describes the various ways in which the target is reached. In every behavior each sub-maneuver has a priority value, at each cycle a dedicated component, called Mission Manager, as explained in section III will execute a safe sub-maneuver with the highest priority; if none of the sub-maneuvers is judged as safe the whole behavior is considered unsafe and than disabled, i.e. will not be proposed as available to the driver.

In table I the main behaviors and sub-maneuvers that compose the Digital Driving paradigm are described. The hierarchical division into behaviors and sub-maneuvers results into a very compact and complete maneuvers catalogue and at the same time gives a strong modularity to the architecture because it allows the reuse of a sub-maneuver across multiple behaviors.

Here a brief description of the behaviors cited in table I is given:

- Urban Mode: this behavior can be used during a trip on urban road, with a single lane, or during an off-road trip. This behavior comprises the possibility to overtake another vehicle, to check and respect traffic lights and roundabout.

- Highway Mode: highway driving behavior, with the possibility to perform lane changes and overtakings.

- Vehicle Follower: a specialized behavior dedicated to the leader-follower paradigm, with this behavior an intelligent vehicle (follower) can follow another vehicle (leader) observing the traffic rules and avoiding obstacles on the path.

- Stop\&Go: a behavior specialized in the management of queue situations in which the intelligent vehicle choose to follow the vehicle ahead at low speeds and short distance, without the ability to see horizontal road sign due to heavy traffic obstructing the lane markings.

- CrossRoad Selection: a behavior that manages all intersections types and allows to change destination in every kind of road.

- Parking: a behavior dedicated to the action of parking the intelligent vehicle; this behavior is intended not as "Park here, now" but as "Park as soon as possible, while driving normally avoiding obstacles and respecting traffic rules".

Each sub maneuver is an implementation of the base submaneuver structure, which consists of the following components:

- Pre-Conditions: List of conditions necessary for the approval of the sub-maneuver;

- Post-Conditions: List of conditions that define the end of the maneuver;

- Exit-Conditions: List of conditions caused by events that are not related to the vehicle, that force the exit from the current maneuver;

- Gate List: list of gates that the vehicle must cross;

The gate concept is the basic idea of this level of planning: a gate integrates information about speed, position and orientation that the vehicle must have in the future trajectory without directly plan an entire trajectory. Basically, a gate is a kind of checkpoint planned in the future trajectory through which the vehicle must pass and it consists of the following components:

- Position and Orientation: $x, y$ and $\alpha$ of the gate in the world frame of reference, a key aspect is that the vehicle must pass the gate with the same orientation of the gate itself;

- Dimension: gate width identifies the maximum allowed error crossing the gate;

- Max Speed and Suggested Speed: vehicle max speed and vehicle suggested speed through the gate, the Low Level Planner will use the suggested speed to plan the speed profile of the vehicle, without exceed the Max Speed;

The gates are planned at a given distance one from each other and the planning step is one of the configuration parameters common to all the sub-maneuvers. The other configuration parameters are the maximum suggested speed difference between two consecutive gates and the maximum orientation difference between two consecutive gates. These last two values are used to avoid that the corresponding parameters (Suggested Speed and $\alpha$ ) calculated by the planner exceed the maximum values achievable by the Low Level Planner. To better present the sub-maneuver structure and the gate concept we present, as an example, the sub-maneuver called "Overtake" in table I, which has the purpose of manage the overtake maneuver during highway driving. The aim of this maneuver is to change lane and overtake the vehicle, without returning in the right lane because in the "Highway Mode" behaviour the vehicle take always the right-hand lane free.

- Preconditions:

- $S_{v}<S_{\text {sugg }}+$ th where $S_{v}$ is the vehicle actual speed, $S_{\text {sugg }}$ the speed suggested by the actual maneuver and th a threshold value;

- Left Lane free (Output of Blind Spot Monitor System);

- Post-Conditions: 


\begin{tabular}{|c|c|c|c|c|c|c|}
\hline & Urban Mode & Highway Mode & Vehicle Follower & Stop\&Go & Crossroad Selection & Parking \\
\hline LKS + ACC & * & ** & & & & \\
\hline Offroad Driving & $*$ & & & & & \\
\hline Ditch Keeping & $*$ & $*$ & & & & \\
\hline Wall Following & $*$ & & & & & \\
\hline GPS Follower & $*$ & & & & & \\
\hline Freespace driving & $*$ & $*$ & $*$ & $*$ & $*$ & $*$ \\
\hline Overtake & $*$ & $*$ & $*$ & & & \\
\hline Lane Changer & & $*$ & $*$ & & & \\
\hline Vehicle Following & & & & $*$ & & \\
\hline Roundabout Intersection & & & & & $*$ & \\
\hline T Intersection & & & & & $*$ & \\
\hline Highway Entrance/Exit & & & & & $*$ & \\
\hline Traffic Light & $*$ & & & & $*$ & \\
\hline Angled Parking & & & & & & $*$ \\
\hline Parallel Parking & & & & & & $*$ \\
\hline
\end{tabular}

TABLE I

BEHAVIORS AND MANEUVERS.

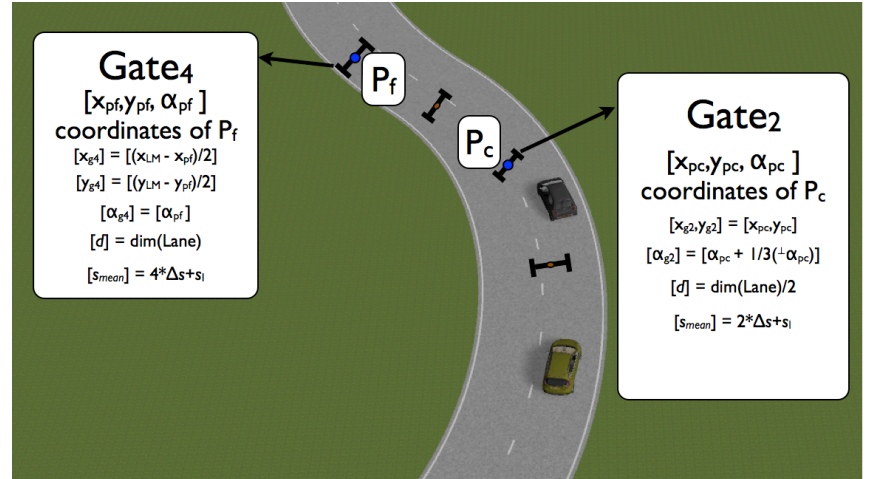

Fig. 2. Overtake submaneuver, the first car is the semi autonomous one, the second represent the vehicle to overtake; the blue dots represent the reference points $P_{c}$ and $P_{f}$, the gates are represented in dark

- Distance from front vehicle $<-L+t h$, where $L$ is the vehicle length;

- $S_{v}>S_{o v}$ where $S_{o v}$ is the speed of the vehicle to overtake;

- Exit Conditions: $S_{o v}>$ MaxSpeed

An example of how gates are determined for a general overtake maneuver is depicted in figure 2. First, two reference points is taken, named $P_{c}$ and $P_{f}$ in the figure. $P_{c}$ is the points in which the vehicle will cross the lane marking and $P_{f}$ is the point at wich the vehicle will be entirely in the left lane. $[x, y, \alpha]$ of the 4 gates are derived directly from these reference points as described in figure 2 . The suggested speed of the gates is gradually increased to reach the target speed in the last gate.

A key aspect of this level of planning is that it doesn't deal with obstacle and with their trajectory since the function of obstacle avoidance is performed by the Low Level Planner.

\section{NeW ARChitecture}

The concepts of vehicle-driver cooperation, described in the last section, has been considered and included in the development of a new system architecture for an intelligent vehicle, with the aim of being able to handle all levels of automations showed in figure 1. This architecture is the final step of "Open intelligent systems for Future Autonomous Vehicles" (OFAV) project. The project objective is the development of an open architecture for future autonomous vehicles to become a standard platform shared by car makers in the design of next generation intelligent vehicles. It is based on a 360 degrees sensorial suite which includes perceptual and decision making modules, with the ultimate goal of providing a vehicle with autonomous driving capabilities and/or supervise the driver's.

The guidelines of the new system architecture, depicted in figure 3, are:

- Introduction of Driver-Vehicle Cooperation: the architecture has been explicitly designed to provide cardriver interaction and cooperation, in particular the planning and decision blocks of the architecture has been designed to allow and ease the integration between differents automation levels and to handle driver interaction with the intelligent vehicle. Plus, a catalogue of maneuvers that would be compatible with both the fully autonomous level and the digital driving level has been designed, as explained in section ??;

- Separation of perception, planning and actuation: as showed in figure 3, percept-plan-act loops has been split in three totally separated levels. This separation leads to many advantages, including detection redundancy reduction, an easier programming of each task and the possibility to perform an intensive testing of each component within the three levels.

- Use of maps in long-term planning: GPS maps has been introduced as cartographic source for long-term planning; in particular maps from the OpenStreetMap (OSM) project has been used: OSM is a collaborative project to create a free editable map of the world [17]. To handle the problem of long distance path planning with real time constraints, a hierarchical approach to the planning problem has been used, in which we three different abstraction levels can be considered: Navigation, Maneuver and Control Level as defined in 


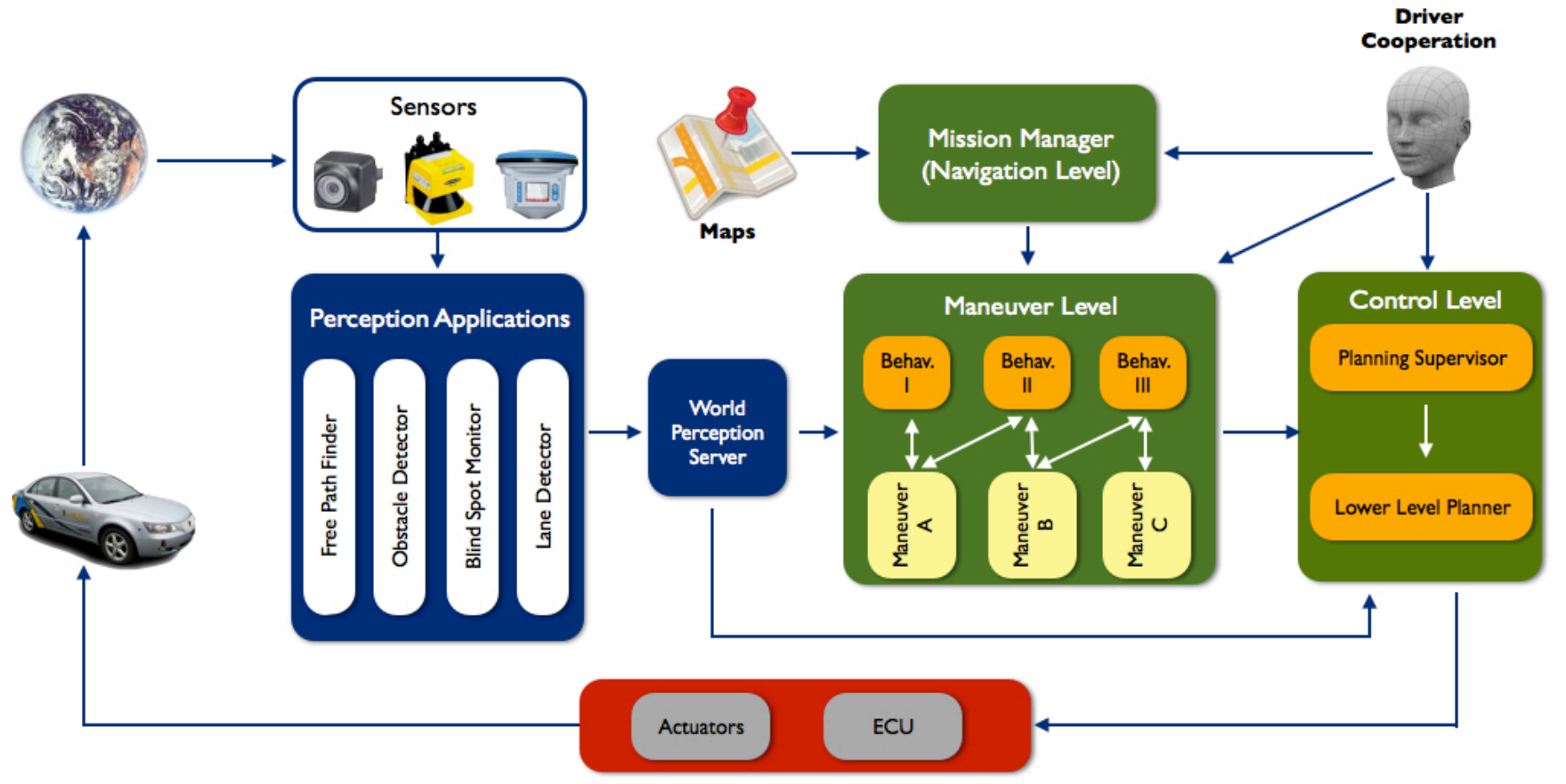

Fig. 3. System architecture implemented for the OFAV project.

Fig.3.

The architecture has been designed to obtain a full integration between all the automation steps of figure 1, thus the software components used during a digital driving session are the same used during a fully automatic session. In general, all the components of a lower level are used from an higher one by means of a software manager that replaces the human driver in that task; for example an intelligent agent, called Mission Manager in figure 3, has been designed covering different functionalities in each level. In case of fully automatic session the Mission Manager serves as high level planner, with the aim to define a route from the start to the destination and to engage at the right time all the maneuvers needed to travel. Otherwise, in case of digital driving session it serves as intelligent driver assistant, with the aim to communicate to the driver feasible maneuvers at each time.

A conflicts resolution policy has been implemented in this architecture; as depicted in figure 3, the driver can interact with the vehicle across all the hierarchal levels; moreover, the driver always has the final decision. The HMIs related to the different automation levels are always active and can be used by the driver to override the Mission Manager behavior: for example is possible to overtake a vehicle even if the Mission Manager judges the action as unsafe simply by taking the wheel and act on the car low level controls (steering wheel and pedals).

\section{TESTING}

In 2010 VisLab made the VisLab Intercontinental Autonomous Challenge (VIAC) [18] [19]. VIAC was the first autonomous driving test on a $13000 \mathrm{~km}$ long extreme route, including all sort of traffic, weather conditions, road infrastructures, and even off-road to really assess the performance of the systems. Another noticeable outcome was the collection of a huge amount of sensors data throughout the journey, providing a testbed for the validation of current and future algorithms. To complete the project, four electricpowered Piaggio Porter minivans, equipped with industrial sensors and computing hardware providing the autonomous driving technology has been leveraged. Great care was taken to ensure that the devices installed on the vehicles did not affect the vehicle performance: sensors, processing systems, and actuation devices were all powered by solar energy, in order not to drain anything from the vehicles standard batteries. This extensive test allowed a thorough test of the systems and technologies, including the robustness of the hardware solutions; the results of the project are summarized in [6].

VIAC was the opportunity to perform a first preliminary test of the Digital Driving paradigm, because during the challenge some systems and functionalities of the new architecture were tested: leader-follower, stop\&go, vehicle detector, waypoint following, panoramic viewing functionality, ditch\&berms detector, and lane and obstacles detector. This project also allowed a deep study of all driving situations, and thanks to the $13,000 \mathrm{~km}$ of driving in an uncontrolled environment it was possible to verify and complete the maneuvers catalog so that it could cover all areas in which a vehicle may pass; the result of this analysis is the maneuvers catalogue summarized in table I. Another important output of the VIAC project is that even with a limited number 
of maneuvers (in VIAC only a subset of the maneuvers of table I was implemented) there were a large number of maneuver change actions, even when the driving conditions was stable, as for example during highway driving; moreover the maneuvers used were strongly linked to the mission that was taking place at that moment. The analysis of the maneuvers used and the analysis of the frequency of their exchange during VIAC lead to the splitting of the maneuver level into two sublayers, as depicted in figure 3, in order to group the maneuvers in functional groups, called behaviors, that would limit the number of driver interventions on the HMI. The last outcome of this test was a study of the use of the HMI that lead to a new HMI that will be used in the Digital Driving interface. Tests and simulations of the Digital Driving have been conducted using Prescan ${ }^{\circledR}$ software.

\section{Conclusions}

In this paper a new vehicle guidance paradigm has been presented toghether with a new system architecture able to support different levels of automation, from manually to fully autonomous driving. The key idea of this paradigm is the possibility to drive a car by simply choosing a behavior proposed by the vehicle itself, without directly interacting with the car's low level controls. One of the key aspect of the proposed implementation of the paradigm is the concept of gate, which is a fast and efficient way to perform a maneuver level planning; the main advantage of the gate concept is that provide a standard method to describe a maneuver without directly involving the planning of a complete trajectory. Another important charateristic is represented by the division of the maneuver level in two sub layers that allows a complete and compact maneuvers catalogue for the digital driving paradigm; in particular the compactness of the catalogue is very important to improve the usability and the user acceptance of the proposed paradigm.

The user acceptance will have a huge effect on the success of Digitad Driving and at a first glance it may be doubetable because it represent kind of driving very different from the traditional one. But the large acceptance of the newest commercial ADAS, like ACC or other lane keeping system, together with the use in the paradigm of a behavioral division of maneuver, as seen in section II, will lead in our opinion to a broad acceptance of the paradigm. However, to test and improve the user acceptance, in the next year a simulator study on the interaction between driver and vehicle will be performed with a large group of partecipants.

The purpose of this paper was to give an overview of the current state of development of a maneuver based driving paradigm and of the state of development of the OFAV project. The next steps in the project are the implementation of another demo test in Parma, in which the new architecture and the digital driving implementation will be completed and tested and a simulator study to test user acceptance of the $\mathrm{HMI}$ and of the maneuver based driving.

\section{ACKNOWLEDGMENTS}

This project was carried out in the frame of the ERC OFAV Grant received by Prof. Alberto Broggi. Simulations and tests have been conduced using PreScan ${ }^{\circledR}$ software provided by TASS.

\section{REFERENCES}

[1] M. Kauer, M. Schreiber, and R. Bruder, "How to conduct a car? a design example for maneuver based driver-vehicle interaction," in Intelligent Vehicles Symposium (IV), 2010 IEEE, june 2010, pp. 1214 -1221 .

[2] A. Broggi, P. Cerri, S. Ghidoni, P. Grisleri, and H. G. Jung, "A New Approach to Urban Pedestrian Detection for Automatic Braking," IEEE Trans. on Intelligent Transportation Systems, vol. 10, no. 4, pp. 594-605, Dec. 2009, ISSN: 1524-9050.

[3] M. Felisa and P. Zani, "Robust monocular lane detection in urban environments," in Procs. IEEE Intelligent Vehicles Symposium 2010, San Diego, CA, USA, June 2010, pp. 591-596.

[4] Y.-L. Chen, V. Sundareswaran, C. Anderson, A. Broggi, P. Grisleri, P. P. Porta, P. Zani, and J. Beck, "TerraMax: Team Oshkosh Urban Robot," Journal of Field Robotics, vol. 25, no. 10, pp. 841-860, Oct. 2008.

[5] D. Braid, A. Broggi, and G. Schmiedel, "The TerraMax Autonomous Vehicle," Journal of Field Robotics, vol. 23, no. 9, pp. 693-708, Sept. 2006.

[6] M. Bertozzi, L. Bombini, A. Broggi, M. Buzzoni, E. Cardarelli, S. Cattani, P. Cerri, A. Coati, S. Debattisti, A. Falzoni, R. I. Fedriga, M. Felisa, L. Gatti, A. Giacomazzo, P. Grisleri, M. C. Laghi, L. Mazzei, P. Medici, M. Panciroli, P. P. Porta, P. Zani, and P. Versari, "VIAC: an Out of Ordinary Experiment," in Procs. IEEE Intelligent Vehicles Symposium 2011, Baden Baden, Germany, June 2011, pp. $175-180$.

[7] M. Bertozzi, L. Bombini, A. Broggi, P. Grisleri, and P. P. Porta, "Camera-Based Automotive Systems," in Smart Cameras, A. N. Belbachir, Ed. Springer US, Oct. 2009, pp. 319-338, ISBN: 9781-4419-0952-7.

[8] C. Urmson et al., "Autonomous driving in urban environments: Boss and the urban challenge," Journal of Field Robotics Special Issue on the 2007 DARPA Urban Challenge, Part I, vol. 25, no. 8, pp. 425-466, June 2008.

[9] S. Geyer, S. Hakuli, H. Winner, B. Franz, and M. Kauer, "Development of a cooperative system behavior for a highly automated vehicle guidance concept based on the conduct-by-wire principle," in Intelligent Vehicles Symposium (IV), 2011 IEEE, june 2011, pp. $411-416$.

[10] United Nations, "Convention on road traffic and road signs," November 1968, vienna, Austria.

[11] O. Flemisch, A. Adams, S. R. Conway, K. H. Goodrich, M. T. Palmer, and P. C. Schutte, "Nasa/tm2003-212672 the h-metaphor as a guideline for vehicle automation and interaction," 2003.

[12] M. Kauer, M. Schreiber, and R. Bruder, "How to conduct a car? a design example for maneuver based driver-vehicle interaction," in Intelligent Vehicles Symposium (IV), 2010 IEEE, june 2010, pp. 1214 -1221 .

[13] Alberto Broggi, "High levels of automation in next generation vehicles," Keynote at the IEEE Intelligent Vehicle Symposium, June 2009.

[14] E. Donges, "A two-level model of driver steering behavior," Human Factors, vol. 20, no. 6, pp. 691-707, 1978.

[15] G. S. H.-H. Nagel, W. Enkelmann, "Fhg-co-driver: From map-guided automatic driving by machine vision to a cooperative driver support," Mathematical and Computer Modelling, vol. 22, no. 47, pp. 185 212, 1995.

[16] M. Schreiber, M. Kauer, and R. Bruder, "Conduct by wire - maneuver catalog for semi-autonomous vehicle guidance," in Intelligent Vehicles Symposium, 2009 IEEE, june 2009, pp. $1279-1284$.

[17] O. Foundation, "Openstreetmap," available at http: //www.openstreetmap.org.

[18] "Vislab intercontinental autonomous challenge," http://en.wikipedia.org/wiki/VIAC.

[19] A. Broggi, P. Cerri, M. Felisa, M. C. Laghi, L. Mazzei, and P. P. Porta, "The VisLab Intercontinental Autonomous Challenge: an Extensive Test for a Platoon of Intelligent Vehicles," Intl. Journal of Vehicle Autonomous Systems, special issue for 10 ${ }^{\text {th }}$ Anniversary, 2011. 